\title{
Reviewers - Volume 24
}

\begin{tabular}{|c|c|}
\hline Samantha Bernstein- & University of Southern \\
\hline Sierra & California \\
\hline \multirow[t]{2}{*}{ Robert Bringle } & Indiana University Purdue \\
\hline & University at Indianapolis \\
\hline Beth Catlett & DePaul University \\
\hline Patti Clayton & Independent Scholar \\
\hline Thomas Deans & University of Connecticut \\
\hline Diane Doberneck & Michigan State University \\
\hline Lina Dostilio & University of Pittsburgh \\
\hline Michelle Dunlap & Connecticut College \\
\hline Joseph Erickson & Augsburg College \\
\hline Janet Eyler & Vanderbilt University \\
\hline Peter Felten & Elon University \\
\hline Ryan Fukumori & Independent Scholar \\
\hline Andy Furco & University of Minnesota \\
\hline Sherril Gelmon & Portland State University \\
\hline \multirow[t]{2}{*}{ Dwight Giles } & University of \\
\hline & Massachusetts-Boston \\
\hline David Greene & Colorado State University \\
\hline \multirow[t]{2}{*}{ Thomas Hahn } & Indiana University Purdue \\
\hline & University at Indianapolis \\
\hline \multirow[t]{2}{*}{ Susan Harris } & University of Southern \\
\hline & California \\
\hline \multirow[t]{2}{*}{ Julie Hatcher } & Indiana University Purdue \\
\hline & University at Indianapolis \\
\hline Michael Hatcher & Valparaiso University \\
\hline Barbara Holland & Independent Scholar \\
\hline Barbara Jacoby & University of Maryland \\
\hline \multirow[t]{2}{*}{ Audrey Jaeger } & North Carolina State \\
\hline & University \\
\hline \multirow[t]{2}{*}{ Emily Janke } & University of North Carolina \\
\hline & Greensboro \\
\hline Susan Jones & The Ohio State University \\
\hline Cathy Jordan & University of Minnesota \\
\hline \multirow[t]{2}{*}{ Arthur Keene } & University of Massachusetts- \\
\hline & Amherst \\
\hline Novella Keith & Temple University \\
\hline \multirow[t]{2}{*}{ Adrianna Kezar } & University of Southern \\
\hline & California \\
\hline
\end{tabular}

\begin{tabular}{|c|c|}
\hline Richard Kiely & Cornell University \\
\hline Lori Kniffin & $\begin{array}{l}\text { University of North Carolina- } \\
\text { Greensboro }\end{array}$ \\
\hline Kelly Lockeman & $\begin{array}{l}\text { Virginia Commonwealth } \\
\text { University }\end{array}$ \\
\hline Sable Manson & $\begin{array}{l}\text { University of Southern } \\
\text { California }\end{array}$ \\
\hline Sam Marullo & Wesley Seminary \\
\hline Ellen Middaugh & San Jose State University \\
\hline A.T. Miller & Cornell University \\
\hline Tania Mitchell & University of Minnesota \\
\hline Barbara Moely & Tulane University \\
\hline Emily Morrison & George Washington University \\
\hline Keith Morton & Providence College \\
\hline Katrina Norvell & Roger Williams University \\
\hline Kerry Ann O’Meara & University of Maryland \\
\hline Andrew Pearl & University of North Georgia \\
\hline Jake Peters & $\begin{array}{l}\text { University of Southern } \\
\text { California }\end{array}$ \\
\hline Kenneth Reardon & $\begin{array}{l}\text { University of } \\
\text { Massachusetts-Boston }\end{array}$ \\
\hline Roger Reeb & University of Dayton \\
\hline Susan Reed & DePaul University \\
\hline Nora Pillard Reynolds & Haverford College \\
\hline John Saltmarsh & $\begin{array}{l}\text { University of Massachusetts- } \\
\text { Boston }\end{array}$ \\
\hline Dan Sarofian-Butin & Merrimack College \\
\hline Si Shen & $\begin{array}{l}\text { University of Southern } \\
\text { California }\end{array}$ \\
\hline Sarah Stanlick & Lehigh University \\
\hline Timothy Stanton & Stanford University \\
\hline Alan Steinberg & Rice University \\
\hline Randy Stoecker & University of Wisconsin \\
\hline Kelly Ward & Washington State University \\
\hline Kathleen Maas Weigert & Loyola University Chicago \\
\hline Rachael Wendler Shah & $\begin{array}{l}\text { University of Nebraska- } \\
\text { Lincoln }\end{array}$ \\
\hline Edward Zlotkowski & Bentley University \\
\hline
\end{tabular}

